\title{
High-resolution grazing incidence small angle x-ray scattering: Investigation of micrometer sized structured polymer films
}

\author{
P. Müller-Buschbaum, E. Bauer, V. Körstgens, J. Wiedersich', S.V. Roth², R. Gehrke² \\ TU München, Physik Department LS E13, James-Franck-Str.1, 85748 Garching (Germany) \\ ${ }^{1}$ TU München, LS Physik Weihenstephan, Vöttinger Strasse 40, 85350 Freising (Germany) \\ ${ }^{2}$ HASYLAB at DESY, Notkestr. 85, 22603 Hamburg (Germany)
}

The structure of polymer surfaces and thin polymeric films at the mesoscopic scale is of interest, both for application and basic research [1]. As the size of many technological devices decreases, the natural length scales of many typical polymers such as the radius of gyration, the persistence length, or the domain size in block copolymers, match the feature size and thus the materials are expected to display a new behaviour $[2,3]$. On the other hand, the tendency towards spontaneous structure formation may stabilize or even generate morphological features of larger size $[4,5]$. As a consequence, the range of interesting polymeric structures starts on the molecular level and extends up to several micrometers. With the ongoing improvement of sample preparation techniques, these structures may extend to macroscopic surface areas of several tens of square centimetres [6]. Along this line of enlarged structured surface areas, very local spatial probes such as atomic force microscopy (AFM) and transmission electron microscopy (TEM) are of less statistical significance. With AFM and TEM only a few exemplary spots of the surface can be probed. The resulting low statistics include the danger of being mislead by an artificial local structure as for example caused by defects. The use of a strongly averaging experimental technique such as scattering is therefore very advantageous [7].

One particular strength of GISAXS is the probing of small sized structures such as micro-phase separation structures in copolymer films. Such molecular structures are easily detected with a relaxed resolution [7]. Following the same principles as in the transmission geometry (SAXS to USAXS) larger length scales may be addressed by an increase in the sample detector distance. In accordance to the transmission this sometimes yields a change in the used abbreviation from GISAX to GIUSAXS (or $x$-ray reflection ultra small-angle scattering in pioneering work [8]). Highly collimated $x$-ray beams enable the extension of GISAXS experiments into the highresolution regime. By making use of the absence of any beamstop, the resolution can be optimised to the detector pixel size as demonstrated in this investigation. With the ongoing development towards high-resolution set-ups large sized surface structures such as phase separation structures in polymer blend films are improvingly determinable. In addition, this development enables the overlap with optical techniques combined with the very high sensitivity of the grazing incidence geometry. However, the high demand on collimation requires the use of high flux sources of large scale facilities such as special synchrotron radiation beamlines.

The model system used consists of blend films of polystyrene (PS) and poly-n-butylacrylate (PnBA) with molecular weights $\mathrm{M}_{\mathrm{w}}=207 \mathrm{k}\left(\mathrm{M}_{\mathrm{w}} / \mathrm{M}_{\mathrm{n}}=1.02\right)$ and $\mathrm{M}_{\mathrm{w}}=260 \mathrm{k}\left(\mathrm{M}_{\mathrm{w}} / \mathrm{M}_{\mathrm{n}}=3.78\right)$, respectively. Both components were blended in toluene solution at different weight ratios of PS:PnBA= 0.1:9.9 to 9:1 and prepared from solution casting onto pre-cleaned silicon (100) substrates. Varying the solution concentration as well as the amount of solution deposited on the solid support enables the installation of different polymer film thicknesses. The smooth drying process in a special designed sample chamber at ambient conditions results in polymer blend films with marked surface structures due to phase separation (the polymer-polymer interaction parameter of PS and PnBA is 0.162 at $20^{\circ} \mathrm{C}$ ). The grazing incidence small angle $\mathrm{x}$-ray scattering (GISAXS) measurements were performed at the BW4 USAX beamline of the DORIS III storage ring at HASYLAB/DESY in Hamburg. The selected wavelength was $0.138 \mathrm{~nm}$. The scattered intensity was recorded with a two dimensional detector which consists of a 2048 x 2048 pixel array. Due to the sample-detector distance of $12.9 \mathrm{~m}$ a high resolution was achieved. The beam divergence in and out of the plane of reflection was set by two entrance cross-slits. At one fixed angle of incident the two-dimensional intensity distribution can be cut in several vertical and horizontal slices with respect to the sample surface. Vertical slices contain mainly scattering information from structures 
perpendicular to the sample surface, whereas horizontal slices contain only scattering contributions with an in-plane information.
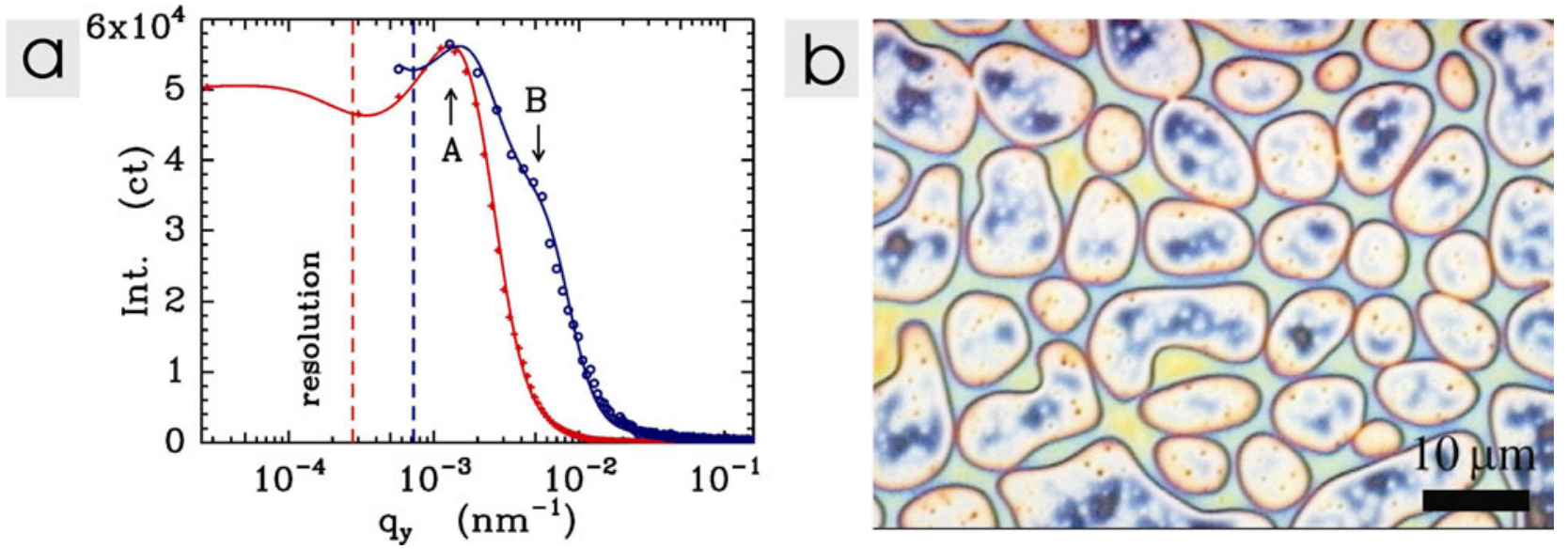

Figure 1: a) Example of blend film sample measured under two different experimental resolution conditions: high resolution set-up (blue open circles) and ultra-high resolution (red crosses). The corresponding resolution limits are marked by vertical lines and the most prominent in-plane length of 4.8 um is marked by an arrow ' $A$ '. The solid lines are fits based on a model described in the text. $b$ ) Corresponding optical micrograph.

In figure 1a GISAXS data measured with the high resolution set-up (blue open circles) are compared with an ultra-high resolution set-up (red crosses). This further increase in resolution marks the actual technical limit with the currently available settings at the BW4 beamline. A further drastic optimisation of the slit settings and the focussing conditions together with a $2 \mathrm{~d}$ dimensional detector with $78 \mu \mathrm{m}$ pixel size only are the base of this improvement. The nominal resolution is $\Delta \mathrm{q}_{\mathrm{y}}=2.75^{*} 10^{-4} \mathrm{~nm}^{-1}$ corresponding to $22 \mu \mathrm{m}$ maximum real space length. This maximum accessible lateral length scale of this ultra-high resolution set-up has been confirmed by the means of Monte-Carlo ray tracing simulation of the beamline BW4 [9].

The structure shown in figure $1 \mathrm{~b}$ is close to the resolution limit of the high resolution set-up and easily detected with the improved resolution. The peak in the intensity (marked with 'A') corresponds to a most prominent in-plane length of $4.8 \mu \mathrm{m}$. It should be noted, that without fitting the data with a simple model, just from the position of the peak in the high resolution set up (blue open circles in figure 1a) a smaller length would be determined, due to the influence of the resolution function. Only in case of a clear separation of the resolution peak and the structural peak, such as measured with the ultra-high resolution set-up for this particular example, the peak position directly gives access to the most prominent in-plane length via a simple Bragg-like analysis and without applying a model fit.

\section{References}

[1] Fedynyshyn T H, Editor (2002) Advances in Resist Technology and Processing XIX Proceedings of SPIE Vol. 4690

[2] Findenegg G H, Herminghaus S (1997) Curr. Opin. Coll. Int. Sci. 2: 301

[3] Russell T P (2002) Science 297: 964

[4] Geoghegan M, Krausch G (2003) Prog. Polym. Sci. 28: 261

[5] Ryan AJ (2002) Nature Materials 1: 8

[6] Müller-Buschbaum P, Bauer E, Wunnicke O, Stamm M (2005) J. Phys. Condens. Matter 17: S363

[7] Müller-Buschbaum P (2003) Anal. Bioanal. Chem. 376: 3

[8] Müller-Buschbaum P, Casagrande M, Gutmann J, Kuhlmann T, Stamm M, Cunis S, von Krosigk G, Lode U, Gehrke R (1998) Europhys. Lett. 42: 517

[9] Roth SV in preparation 